\title{
Elementary excitations and thermodynamics of zig-zag spin ladders with alternating nearest neighbor exchange interactions
}

\author{
A.A. Zvyagin \\ B. Verkin Institute for Low Temperature Physics and Engineering of the National Academy of Sciences of Ukraine \\ 47 Lenin Ave., Kharkov 61103, Ukraine \\ IFW Dresden, Institute for Solid State Research, P.O. 270116, Dresden D-01171, Germany \\ E-mail: zvyagin@ilt.kharkov.ua
}

V.O. Cheranovskii

V.N. Karazin Kharkov National University, 4 Svobody Sq., Kharkov 61077, Ukraine

Received January 12, 2009, revised January 20, 2009

\begin{abstract}
The one-dimensional spin-1/2 model, in which the alternation of the exchange interactions between neighboring spins is accompanied by the next-nearest neighbor (NNN) spin exchange (zig-zag spin ladder with the alternation) is studied. Thermodynamic characteristics of the considered quantum spin chain are obtained in the mean-field like approximation. Depending on the strength of the NNN interactions, the model manifests either the spin-gapped behavior of low-lying excitations at low magnetic fields, or the ferrimagnetic ordering in the ground state with gapless low-lying excitations. The system undergoes second order or first order quantum phase transitions, governed by the external magnetic field, NNN coupling strength, and the degree of the alternation. Hence, NNN spin-spin interactions in a dimerized quantum spin chain can produce a spontaneous magnetization. On the other hand, for quantum spin chains with a spontaneous magnetization, caused by NNN spin-spin couplings, the alternation of nearest-neighbor (NN) exchange interactions can be the reason for destroying of that magnetization and the onset of a spin gap for low-lying excitations. Alternating NN interactions produce a spin gap between two branches of low-energy excitations, and the NNN interactions yield asymmetry of dispersion laws of those excitations, with possible minima, corresponding to incommensurate structures in the spin chain.
\end{abstract}

PACS: $75.10 . P q$ Spin chain models;

75.40.Cx Static properties.

Keywords: spin chain, spin frustration, zig-zag spin ladders.

\section{Introduction}

Last decade the interest in quasi-one-dimensional quantum spin systems has grown considerably. In these systems spin-spin interactions along one space direction are much stronger than couplings along other directions. The interest of physicists to low-dimensional quantum spin systems is motivated, first of all, by the progress in the preparation of substances with well defined one-dimensional subsystems. Another reason for studying quasi-one-dimensional quantum spin systems is the possibility to compare experimental data with exact solutions for one-dimensional models [1]. In one-dimensional systems quantum fluctuations are strongly enhanced due to peculiarities in densities of states. According to the Mermin-Wagner theorem [2], low-dimensional spin systems cannot have magnetic ordering at any nonzero temperature, due to mentioned quantum fluctuations. On the other hand, such systems often manifest quantum phase transitions, which take place in the ground state, and which are governed by other than the temperature parameters, like an external magnetic field, external or internal (caused by chemical substitutions) pressure, etc.

During last years more attention was paid to theoretical studies of one-dimensional quantum spin models with not only nearest-neighbor (NN) spin-spin interactions, 
but also with next-nearest neighbor (NNN) ones. Those models are closer to real quasi-one-dimensional magnets comparing to standard ones with only nearest-neighbor couplings [3]. Such interactions are often present in oxides of transition metals, where the direct exchange between magnetic ions is complimented by the superexchange between magnetic ions via nonmagnetic ones. Some transition metal-oxide compounds exhibiting properties of quasi-one-dimensional spin systems and spinfrustrated systems have been the topic of recent studies because of their large variety of very interesting low temperature properties. The low-energy physics of these systems is mostly determined by a strong exchange interactions between spin-1/2 double chains, formed by edge-sharing $\mathrm{CuO}_{6}$ or $\mathrm{VO}_{6}$ octahedra, in which neighboring and next-neighboring ions of $\mathrm{Cu}^{2+}$ or $\mathrm{V}^{4+}$ with spins $1 / 2$ are surrounded by oxygen ions. Such a configuration of those oxides produces a very special temperature and magnetic behavior of many low energy characteristics of those compounds. For the consistent explanation of several experiments [1] by means of inelastic neutron scattering, optical conductivity and nuclear magnetic resonance one needs to account for relatively large values of NNN spin-spin interactions. Classical counterparts of these models with antiferromagnetic NNN interactions manifest the spin frustration. The classical lowest energy state is highly degenerate [5]. For such models the standard quasi-classical theoretical description based on the quantization of small deviations of classical vectors of magnetization of magnetic sublattices often cannot be applied. This is why, quantum spin models with NNN spin-spin interactions are of principal importance. Also, one-dimensional quantum spin models with NN and NNN spin-spin couplings often manifest quantum phase transitions: several one-dimensional quantum models, belonging to that class, see, e.g., Refs. 6-12, can be solved exactly, using Bethe ansatz. However, exact solutions were obtained for models with additional, perhaps non-realistic spin-spin couplings. Many transition metal compounds, like copper oxides, are believed to reveal features, characteristic for quantum phase transitions. On the other hand, it turns out that some quasi-one dimensional spin systems manifest features, which are characteristic for doubling of magnetic elementary in-chain cells, e.g., the alternation of effective $g$-factors and/or dimerization of the nearestneighbor exchange constants.

In this paper we study a one-dimensional model of quantum spins with $\mathrm{NN}$ and NNN interactions (or, in other words, the spin zig-zag ladder) and dimerization of the NN coupling. The main goal of our work is to construct a theory of a realistic model of a quantum spin chain, which permits to analytically describe quantum phase transitions caused by spin-spin interactions and the external magnetic field. That model, on the one hand, has to contain spin-frustrating NNN spin exchange interactions, which usually produce incommensurate magnetic structures [10]. On the other hand, the model has to have alternating $\mathrm{NN}$ exchange interactions, which can be the reason for the formation of a spin gap for low-lying excitations there [1]. Notice, that antiferromagnetic $\mathrm{NN}$ and NNN interactions themselves also can produce a gap [13]. In recent experiments on quasi-one-dimensional quantum magnets [14] it was pointed out that for some range of parameters it is possible that incommensurate magnetic structures co-exist with the doubling of elementary cells for one-dimensional spin subsystems, see also Ref. 15. The Hamiltonian of our model contains both of mentioned factors. As it will be clear from our results, taking into account these two factors (NNN interactions and dimerization), our theory really reveals quantum phase transitions (governed also by the magnetic field) between magnetic commensurate and/or incommensurate phases, with spin-gapped and gapless excitations.

\section{The model}

The Hamiltonian of the quantum spin model with alternating NN couplings and NNN exchange interactions has the form:

$$
\begin{gathered}
\mathcal{H}=-J_{1} \sum_{n}\left(S_{n, 1}^{x} S_{n, 2}^{x}+S_{n, 1}^{y} S_{n, 2}^{y}\right)-J_{1}^{z} \sum_{n} S_{n, 1}^{z} S_{n, 2}^{z}-J_{2} \sum_{n}\left(S_{n, 2}^{x} S_{n+1,1}^{x}+S_{n, 2}^{y} S_{n+1,1}^{y}\right)-J_{2}^{z} \sum_{n} S_{n, 2}^{z} S_{n+1,1}^{z}+ \\
+J_{N N} \sum_{n}\left(S_{n, 1}^{x} S_{n+1,1}^{x}+S_{n, 1}^{y} S_{n+1,1}^{y}+S_{n, 2}^{x} S_{n+1,2}^{x}+S_{n, 2}^{y} S_{n+1,2}^{y}\right)+J_{N N}^{z} \sum_{n}\left(S_{n, 1}^{z} S_{n+1,1}^{z}+S_{n, 2}^{z} S_{n+1,2}^{z}\right)-H \sum_{n}\left(\mu_{1} S_{n, 1}^{z}+\mu_{2} S_{n, 2}^{z}\right),
\end{gathered}
$$

where $S_{n, 1,2}^{x, y, z}$ are the operators of the spin-1/2 projections of the spin in the $n$th cell, which belongs to the sublattice 1 or $2, \mu_{1,2} \geq 0$ are effective magnetons of the sublattices,
$H$ is the external magnetic field, directed along $z$ axis, $J_{1,2}$ are the alternating exchange coupling constants between $\mathrm{NN}$ spins in the cell and between cells, $J_{1,2}^{z}-J_{1,2}$ is the 
uniaxial magnetic anisotropy of the $\mathrm{NN}$ interaction, $J_{N N}$ is the interaction constant between NNN spins in a chain, and $J_{N N}^{Z}-J_{N N}$ is the uniaxial magnetic anisotropy of the NNN interaction. For the illustration, see Fig. 1.

It is known that the sign of $J_{1}$ and $J_{2}$ usually does not play a role [1]. While for $J_{1}^{z}, J_{2}^{z}<0$ the situation is investigated in detail, e.g., in theoretical and experimental studies devoted to the quasi-one-dimensional spin-1/2 compound $\mathrm{CuGeO}_{3}$, see Ref. 16, the opposite case of positive exchange constants, or with different signs of them is less known, however it is realized in some real quasi-one-dimensional compounds $[3,5]$.

After the Jordan-Wigner transformation, see, e.g., Ref. 1

$$
S_{n, 1,2}^{z}=\frac{1}{2}-a_{n, 1,2}^{\dagger} a_{n, 1,2}=\frac{1}{2} \sigma_{n, 1,2}
$$

$$
\begin{gathered}
S_{n, 1}^{+} \equiv S_{n, 1}^{x}+i S_{n, 1}^{y}=\prod_{m<n} \sigma_{m, 1} \sigma_{m, 2} a_{n, 1}, \\
S_{n, 1}^{-} \equiv S_{n, 1}^{x}-i S_{n, 1}^{y}=a_{n, 1}^{\dagger} \prod_{m<n} \sigma_{m, 1} \sigma_{m, 2}, \\
S_{n, 2}^{+}=\prod_{m<n} \sigma_{m, 1} \sigma_{m, 2} \sigma_{n, 1} a_{n, 2}, \\
S_{n, 2}^{-}=a_{n, 2}^{\dagger} \prod_{m<n} \sigma_{m, 1} \sigma_{m, 2} \sigma_{n, 1},
\end{gathered}
$$

where $a_{n, 1,2}^{\dagger}$ and $a_{n, 1,2}$ are creation and destruction operators, which satisfy fermionic anticommutation relations, the Hamiltonian (1) obtains the form

$$
\begin{gathered}
\mathcal{H}=-\left(J_{1} / 2\right) \sum_{n}\left(a_{n, 1}^{\dagger} a_{n, 2}+\text { H.c. }\right)-\left(J_{1}^{z} / 4\right) \sum_{n}\left(1-2 a_{n, 1}^{\dagger} a_{n, 1}\right)\left(1-2 a_{n, 2}^{\dagger} a_{n, 2}\right)-\left(J_{2} / 2\right) \sum_{n}\left(a_{n, 2}^{\dagger} a_{n+1,1}+\text { H.c. }\right)- \\
-\left(J_{2}^{z} / 4\right) \sum_{n}\left(1-2 a_{n+1,1}^{\dagger} a_{n+1,1}\right)\left(1-2 a_{n, 2}^{\dagger} a_{n, 2}\right)+\left(J_{N N} / 2\right) \sum_{n}\left[a_{n, 1}^{\dagger}\left(1-2 a_{n, 2}^{\dagger} a_{n, 2}\right) a_{n+1,1}+a_{n, 2}^{\dagger}\left(1-2 a_{n+1,1}^{\dagger} a_{n+1,1}\right) a_{n+1,2}+\text { H.c. }\right]+ \\
+\left(J_{N N}^{z} / 4\right) \sum_{n}\left[\left(1-2 a_{n, 1}^{\dagger} a_{n, 1}\right)\left(1-2 a_{n+1,1}^{\dagger} a_{n+1,1}\right)+\left(1-2 a_{n, 2}^{\dagger} a_{n, 2}\right)\left(1-2 a_{n+1,2}^{\dagger} a_{n+1,2}\right)\right]- \\
\quad-(H / 2) \sum_{n}\left[\mu_{1}\left(1-2 a_{n, 1}^{\dagger} a_{n, 1}\right)+\mu_{2}\left(1-2 a_{n, 2}^{\dagger} a_{n, 2}\right)\right] .
\end{gathered}
$$

The Hamiltonian (3) consists of the sum of the quadratic form of Fermi operators, the part, which contains the products of four Fermi operators, which correspond to the pair interactions between fermions, and the part, which does not depend on operators.

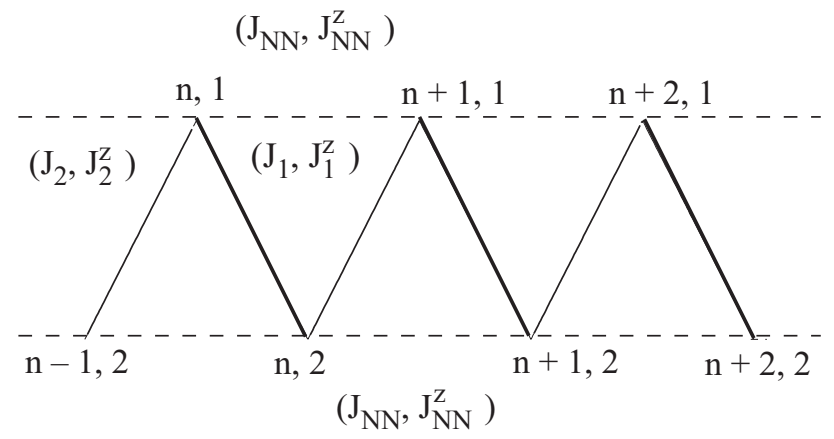

Fig. 1. Illustration of the zig-zag spin ladder. Solid zig-zag lines denote nearest neighbor couplings. Bold line denotes the bonds with the exchange constants $J_{1}$ and $J_{1}^{z}$, the thick one denotes the bonds with coupling constants $J_{2}$ and $J_{2}^{z}$. Dashed lines denote next-nearest neighbor interactions with coupling constants $J_{N N}$ and $J_{N N}^{Z}$.

\section{Mean-field like approximation}

In what follows we use the mean-field like (Hartry-Fock like) approximation. Let us introduce the notations

$$
\begin{gathered}
m_{1,2}^{z}=\frac{1}{2}-\left\langle a_{n, 1,2}^{\dagger} a_{n, 1,2}\right\rangle, \\
\gamma_{1}=\left\langle a_{n .1}^{\dagger} a_{n, 2}\right\rangle=\left\langle a_{n, 2}^{\dagger} a_{n, 1}\right\rangle, \\
\gamma_{2}=\left\langle a_{n+1,1}^{\dagger} a_{n, 2}\right\rangle=\left\langle a_{n, 2}^{\dagger} a_{n+1,1}\right\rangle, \\
\gamma_{N N 1,2}=\left\langle a_{n, 1,2}^{\dagger} a_{n+1,1,2}\right\rangle=\left\langle a_{n+1,1,2}^{\dagger} a_{n, 1,2}\right\rangle,
\end{gathered}
$$

where brackets denote the thermodynamic average, either with the ground state wave function for zero temperature, $T=0$, or with the density matrix for nonzero temperatures. Using the Wick's theorem and the mean field-like approximation we can re-write the Hamiltonian (3) as 


$$
\begin{gathered}
\mathcal{H} \approx \sum_{n}\left[a_{n, 1}^{\dagger} a_{n, 1}\left(\mu_{1} H+J_{1}^{z} m_{2}^{z}+J_{2}^{z} m_{1}^{z}-2 J_{N N}^{z} m_{1}^{z}-2 J_{N N} \gamma_{N N 2}\right)+\right. \\
+a_{n, 2}^{\dagger} a_{n, 2}\left(\mu_{2} H+J_{2}^{z} m_{1}^{z}+J_{1}^{z} m_{2}^{z}-2 J_{N N}^{z} m_{2}^{z}-2 J_{N N} \gamma_{N N 1}\right)+\left(a_{n, 1}^{\dagger} a_{n, 2}+a_{n, 2}^{\dagger} a_{n, 1}\right) \times \\
\times\left[-\left(J_{1} / 2\right)+J_{1}^{z} \gamma_{1}+2 J_{N N} \gamma_{2}\right]+\left(a_{n+1,1}^{\dagger} a_{n, 2}+a_{n, 2}^{\dagger} a_{n+1,1}\right)\left[-\left(J_{2} / 2\right)+J_{2}^{z} \gamma_{2}+2 J_{N N} \gamma_{1}\right]+ \\
\left.+\left(a_{n, 1}^{\dagger} a_{n+1,1}+a_{n+1,1}^{\dagger} a_{n, 1}\right) \times\left(J_{N N} m_{2}^{z}-J_{N N}^{z} \gamma_{N N 1}\right)+\left(a_{n, 2}^{\dagger} a_{n+1,2}+a_{n+1,2}^{\dagger} a_{n, 2}\right) \times\left(J_{N N} m_{1}^{z}-J_{N N}^{z} \gamma_{N N 2}\right)\right]+C
\end{gathered}
$$

where the last term is the part, which does not contain operators. Naturally, the mean field-like approximation does not take into account correctly interactions between excitations (for example, it does not take into account bound states), but it qualitatively describes the possibility of quantum phase transitions in the studied model. After the Fourier transform

$$
a_{n, 1,2}=N^{-1 / 2} \sum_{k} a_{k, 1,2} \mathrm{e}^{i k n}
$$

and similar for $a_{n, 1,2}^{\dagger}$, where $N$ is the number of unit cells, and a unitary transformation this Hamiltonian can be diagonalized

$$
\mathcal{H}=\sum_{k} \sum_{j=1}^{2} \varepsilon_{k, j} b_{k, j}^{\dagger} b_{k, j}+C
$$

where

$$
\varepsilon_{k, 1,2}=\frac{1}{2}\left[A_{1} \pm \sqrt{B_{1}^{2}+B_{2}^{2}+2 B_{1} B_{2} \cos (k)+A^{2}}\right]
$$

and

$$
\begin{aligned}
A_{1}=\left[\left(\mu_{1}+\mu_{2}\right) H+\left(m_{1}^{z}+m_{2}^{z}\right)\left(J_{1}^{z}+J_{2}^{z}-2 J_{N N}^{z}\right)-2\left(\gamma_{N N 1}+\gamma_{N N 2}\right) J_{N N}\right]+ & \\
& +2 \cos (k)\left[\left(m_{1}^{z}+m_{2}^{z}\right) J_{N N}-\left(\gamma_{N N 1}+\gamma_{N N 2}\right) J_{N N}^{z}\right] \\
A= & {\left[\left(\mu_{1}-\mu_{2}\right) H-2\left(m_{1}^{z}-m_{2}^{z}\right) J_{N N}^{z}+2\left(\gamma_{N N 1}-\gamma_{N N 2}\right) J_{N N}\right]-} \\
& -2 \cos (k)\left[\left(m_{1}^{z}-m_{2}^{z}\right) J_{N N}+\left(\gamma_{N N 1}-\gamma_{N N 2}\right) J_{N N}^{z}\right] \\
B_{1}= & -J_{1}+2 J_{1}^{z} \gamma_{1}+4 J_{N N} \gamma_{2}, \quad B_{2}=-J_{2}+2 J_{2}^{z} \gamma_{2}+4 J_{N N} \gamma_{1} .
\end{aligned}
$$

The value of the (possible) gap between two bands is determined by

$$
\sqrt{A^{2}+B_{1}^{2}+B_{2}^{2}+2 B_{1} B_{2} \cos (k)} \text {. }
$$

It is then the easy task to obtain thermodynamic characteristics of the model. For example, the free energy (for $T \neq 0$ ) of the quantum alternating spin chain per unit cell is equal to

$$
f=\frac{C}{N}-T \sum_{k} \sum_{j=1}^{2} \ln \left(1+\mathrm{e}^{\frac{-\varepsilon_{k, j}}{T}}\right) .
$$

The $z$-projection of the average magnetization of the system per cell is

$$
M^{z}=\frac{1}{2} \sum_{k} \sum_{j=1}^{2} \frac{\partial \varepsilon_{k, j}}{\partial H} \tanh \left(\frac{\varepsilon_{k, j}}{2 T}\right) .
$$

We can also calculate the magnetic susceptibility per cell 


$$
\chi=\frac{1}{4} \sum_{k} \sum_{j=1}^{2}\left[2 \frac{\partial^{2} \varepsilon_{k, j}}{\partial H^{2}} \tanh \left(\frac{\varepsilon_{k, j}}{2 T}\right)+\frac{1}{T}\left(\frac{\partial \varepsilon_{k, j}}{\partial H}\right)^{2}\left[\cosh \left(\frac{\varepsilon_{k, j}}{2 T}\right)\right]^{-2}\right]
$$

the staggered magnetic susceptibility

$$
\chi_{\text {st }}=\frac{\left(\mu_{1}-\mu_{2}\right)^{2}}{2} \sum_{k} \sum_{j=1}^{2}\left[\frac{\partial^{2} \varepsilon_{k, j}}{\partial\left(\mu_{1}-\mu_{2}\right)^{2} H^{2}} \tanh \frac{\varepsilon_{k, j}}{2 T}+\left(\frac{\partial \varepsilon_{k, j}}{\partial\left(\mu_{1}-\mu_{2}\right) H}\right)^{2} \frac{1}{2 T \cosh ^{2}\left(\varepsilon_{k, j} / 2 T\right)}\right]
$$

and the specific heat

$$
c=\sum_{k} \sum_{j=1}^{2} \frac{\varepsilon_{k, j}^{2}}{4 T^{2} \cosh ^{2}\left(\varepsilon_{k, j} / 2 T\right)}
$$

per unit cell. One can see from the last equation that the temperature dependence of the specific heat can manifest two maxima, related to two kinds of excitations in the system.

The self-consistency conditions for the mean field parameters (1) at nonzero temperature can be written as

$$
\begin{gathered}
m_{1,2}^{z}=\frac{1}{2} \sum_{k}\left(\left[1 \pm \frac{A}{\sqrt{A^{2}+B_{1}^{2}+B_{2}^{2}+2 B_{1} B_{2} \cos (k)}}\right] \tanh \left(\frac{\varepsilon_{k, 1}}{2 T}\right)+\left[1 \mp \frac{B_{1,2}+\cos (k) B_{2,1}}{\sqrt{A^{2}+B_{1}^{2}+B_{2}^{2}+2 B_{1} B_{2} \cos (k)}}\left[\tanh \left(\frac{\varepsilon_{k, 1}}{2 T}\right)-\tanh \left(\frac{\varepsilon_{k, 2}}{2 T}\right)\right]\right.\right. \\
\gamma_{1,2}=\frac{1}{4} \sum_{k} \frac{\left.\tanh \left(\frac{\varepsilon_{k, 2}}{2 T}\right)\right]}{\sqrt{A^{2}+B_{1}^{2}+B_{2}^{2}+2 B_{1} B_{2} \cos (k)}} \\
\gamma_{N N 1,2}=-\frac{1}{2} \sum_{k} \cos (k)\left(\tanh \left(\frac{\varepsilon_{k, 1}}{2 T}\right)\left[1 \mp \frac{A}{\sqrt{A^{2}+B_{1}^{2}+B_{2}^{2}+2 B_{1} B_{2} \cos (k)}}\right]+\right. \\
\left.+\left[1 \pm \frac{A}{\sqrt{A^{2}+B_{1}^{2}+B_{2}^{2}+2 B_{1} B_{2} \cos (k)}}\right] \tanh \left(\frac{\varepsilon_{k, 2}}{2 T}\right)\right)
\end{gathered}
$$

From these equations one can see, in particular, that $\gamma_{1}$ is different from $\gamma_{2}$, if $J_{1} \neq J_{2}$, or if $J_{N N} \neq 0$.

\subsection{High temperature limit}

For high temperatures $\left|\varepsilon_{k, 1,2}\right|<<T$ Eqs. (15) can be transformed to the form

$$
\begin{gathered}
8 T \gamma_{1}=4 J_{N N} \gamma_{2}+2 J_{1}^{z} \gamma_{1}-J_{1}, \\
8 T \gamma_{2}=4 J_{N N} \gamma_{1}+2 J_{2}^{z} \gamma_{2}-J_{2}, \\
2 T\left(m_{1}^{z}+m_{2}^{z}\right)=\left(\mu_{1}+\mu_{2}\right) H+\left(m_{1}^{z}+m_{2}^{z}\right) \times \\
\times\left(J_{1}^{z}+J_{2}^{z}-2 J_{N N}^{z}\right)-2\left(\gamma_{N N 1}+\gamma_{N N 2}\right) J_{N N}, \\
2 T\left(m_{1}^{z}-m_{2}^{z}\right)=\left(\mu_{1}-\mu_{2}\right) H-\left(m_{1}^{z}-m_{2}^{z}\right) 2 J_{N N}^{z}- \\
-2\left(\gamma_{N N 1}-\gamma_{N N 2}\right) J_{N N}, \\
2 T \gamma_{N N 1}=-m_{2}^{z} J_{N N}-2 \gamma_{N N 1} J_{N N}, \\
2 T \gamma_{N N 2}=-m_{1}^{z} J_{N N}-2 \gamma_{N N 2} J_{N N}^{z} .
\end{gathered}
$$

The solution of Eqs. (16) in the main in $1 / T$ approximation is $m_{1,2}^{z} \approx \mu_{1,2} H / T, \gamma_{1,2} \approx-J_{1,2} / 8 T$, and $\gamma_{N N 1,2} \approx$ $\approx \mu_{2,1} H J_{N N} / 2 T^{2}$. Using this solution it is easy to show that $m_{1,2}^{z}$ go to zero with $H$ for nonzero temperatures, in accordance with the Mermin-Wagner theorem. Also, nonzero values of $\gamma_{1,2}$ are determined in the first order in $T^{-1}$ by the NN exchange interaction $J_{1,2}$, while the necessary condition for $\gamma_{N N 1,2} \neq 0$ is the nonzero value of $J_{N N}$, as expected. It is interesting to notice that $\gamma_{N N 1,2} \neq 0$ is connected with the nonzero value of the external magnetic field for high temperatures. It turns out that $\gamma_{N N 1,2}$ become nonzero in higher order in $1 / T$, comparing to $m_{1,2}^{z}$ and $\gamma_{1,2}$. It is also important to notice that in the main approximation at high temperatures $\gamma_{N N 1}=\gamma_{N N 2}$, if $\mu_{1}=\mu_{2}$.

\subsection{The ground state}

The most important properties of the one-dimensional spin system can be, of course, seen in the ground state. The ground state of any fermion system is organized as the total filling of the Fermi-Dirac sea(s), i.e. all fermionic states 
with negative energies are occupied, while all fermionic states with positive energies are empty.

The ground state filling of two fermionic bands Eqs. (8) depends on the relative values of the parameters of the considered model. Let us consider how different terms in Eqs. (8) act. First, one can see from Eqs. (8) and (9) that both bands are symmetric with respect to $k$. Also, it turns out that the term in $A_{1}$, which does not depend on $k$, shifts the positions of both of two bands with respect to zero of the energy axis, i.e. regulates the ground state filling of bands. The term in $A$, which does not depend on $k$, shifts two bands with respect to each other, in particular, its absolute value governs the magnitude of the gap between two bands. Hence, in particular, the external magnetic field, on the one hand, governs the filling of bands, and, on the other hand, the staggered component of the action of the field, caused by the difference $\mu_{1}-\mu_{2}$, changes the value of the gap between bands. Also, the gap is determined by the absolute value of the difference between $B_{1}$ and $B_{2},\left|\gamma_{N N 1}-\gamma_{N N 2}\right|$, and $\left|m_{1}^{z}-m_{2}^{z}\right|$. In particular, for $A=0, B_{1}=B_{2}$, the gap between two bands becomes zero. One can see that the gap is zero if $J_{1}=J_{2}, J_{1}^{z}=J_{2}^{z}$, even for $\mu_{1} \neq \mu_{2}$ for $J_{N N}^{z}=J_{N N}=0$. However, nonzero NNN interactions can also produce a gap even if $J_{1}=J_{2}$, $J_{1}^{z}=J_{2}^{z}$. On the other hand, the terms in $A_{1}$ and $A$, which are proportional to $\cos (k)$, i.e. those, which are caused by nonzero NNN couplings, are responsible for the asymmetry of the bands with respect to each other. Without those terms the bands are symmetric, see, the illustration in Fig. 2. For $m_{1}^{z}=m_{2}^{z}$ and $\gamma_{N N 1}=\gamma_{N N 2}$, if $\left(m_{1}^{z}+m_{2}^{z}\right) J_{N N}<\left(\gamma_{N N 1}+\gamma_{N N 2}\right) J_{N N}^{z}$, the lower band has only one minimum at $k=0$, while the upper band can have one minimum at $k=0$ and two maxima, situated symmetrically with respect to $k=0$, cf. Fig. 3 (see the discussion below). On the other hand, for $m_{1}^{z}=m_{2}^{z}$ and $\gamma_{N N 1}=\gamma_{N N 2}$, if $\left(m_{1}^{z}+m_{2}^{z}\right) J_{N N}>\left(\gamma_{N N 1}+\gamma_{N N 2}\right) J_{N N}^{z}$, the upper band has only one maximum at $k=0$, while the

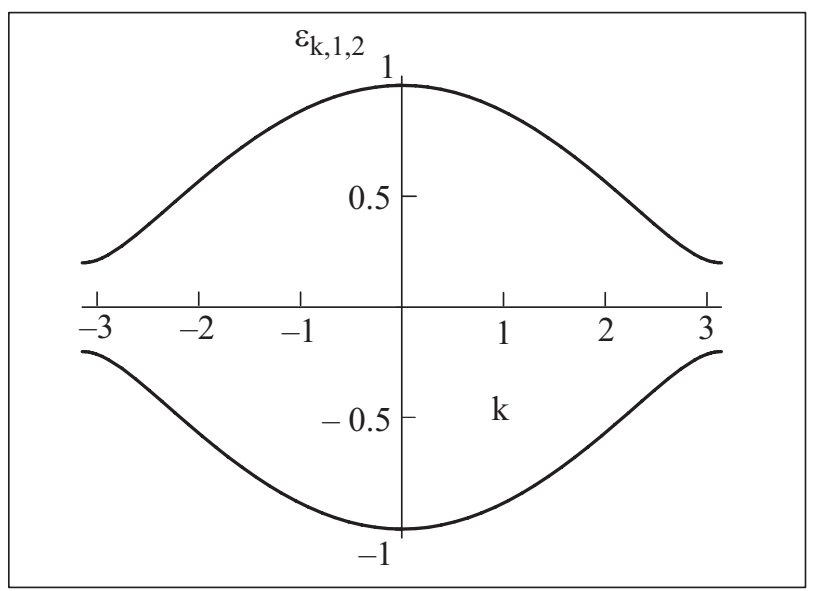

Fig. 2. Symmetric dispersion laws $\varepsilon_{k, 1,2}$ of the studied model (e.g., at $J_{N N}^{z}=J_{N N}=0$ with the nonzero spin gap, caused by $\left.J_{1} \neq J_{2}\right)$.

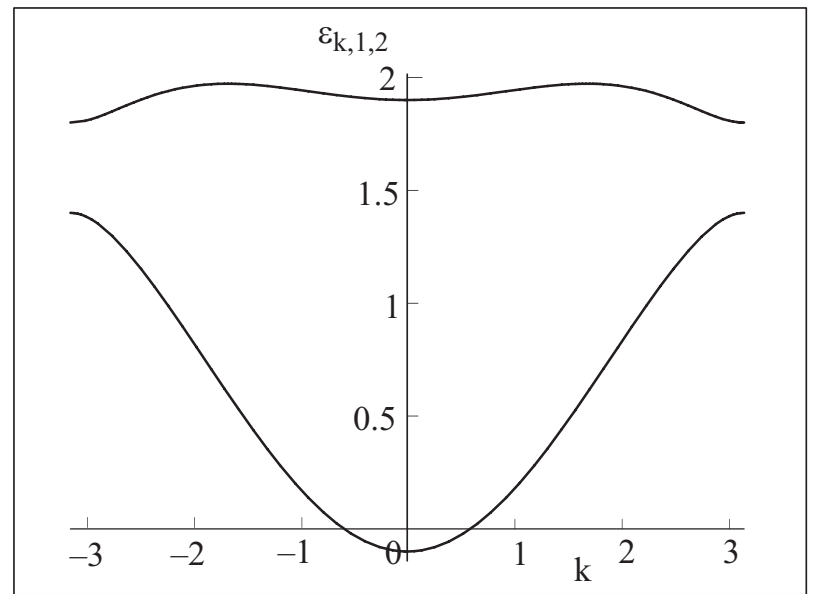

Fig. 3. Asymmetric dispersion laws $\varepsilon_{k, 1,2}$ of the studied model with one minimum of the lower band and two maxima of the upper band. The asymmetry is caused by NNN interactions.

lower band can have one maximum at $k=0$ and two minima, situated symmetrically with respect to $k=0$ point, cf. Fig. 4. Nonzero values of $m_{1}^{z}-m_{2}^{z}$ and $\gamma_{N N 1}-\gamma_{N N 2}$, on the other hand, can produce (being also the gap-yielding reason) several extremal points of both bands (together with $k=0, \pm \pi)$.

In the thermodynamic limit $N \rightarrow \infty$ the self-consistency conditions can be written for the simple case of two Dirac seas, each connected with the upper or lower band, respectively, of Eq. (8), in the ground state as

$$
\begin{gathered}
m_{1,2}^{z}=\frac{1}{2}-\frac{k_{c 1}+k_{c 2}}{2 \pi} \mp \frac{1}{4 \pi}\left[\int_{-k_{c 1}}^{k_{c 1}}-\int_{-k_{c 2}}^{k_{c 2}}\right] d k \times \\
\times \frac{A}{\sqrt{A^{2}+B_{1}^{2}+B_{2}^{2}+2 B_{1 B_{2}} \cos (k)}},
\end{gathered}
$$

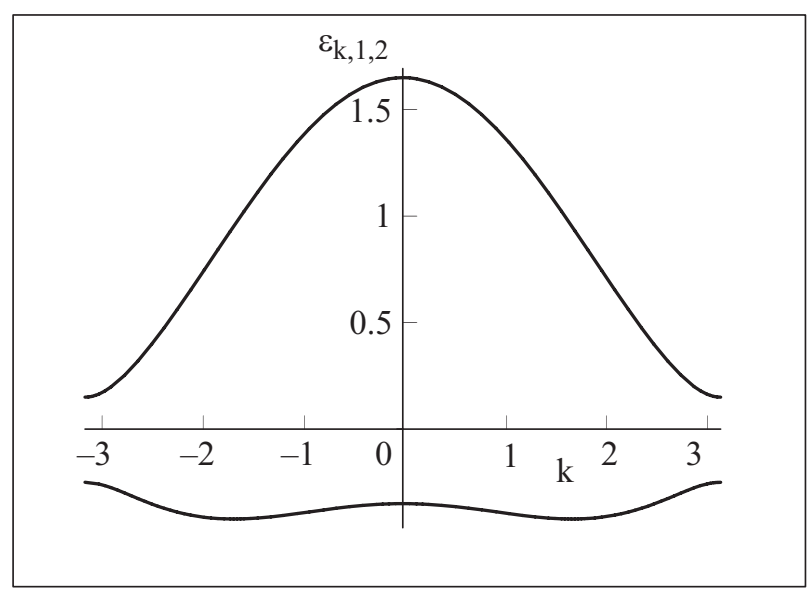

Fig. 4. Asymmetric dispersion laws $\varepsilon_{k, 1,2}$ of the studied model with one maximum of the upper band and two minima of the lower band. 


$$
\begin{gathered}
\gamma_{1,2}=\frac{1}{8 \pi}\left[\int_{-k_{c 1}}^{k_{c 1}}-\int_{k_{c 2}}^{k_{c 2}}\right] d k \frac{B_{1,2}+\cos (k) B_{2,1}}{\sqrt{A^{2}+B_{1}^{2}+B_{2}^{2}+2 B_{1} B_{2} \cos (k)}} \\
\gamma_{N N 1,2}=\frac{1}{2 \pi}\left[\sin \left(k_{c 1}\right)+\sin \left(k_{c 2}\right)\right] \pm \frac{1}{4 \pi}\left[\int_{-k_{c 1}-k_{c 2}}^{k_{c 1}} \int_{c 2}^{k_{c 2}}\right] d k \cos (k) \times \\
\times \frac{A}{\sqrt{A^{2}+B_{1}^{2}+B_{2}^{2}+2 B_{1} B_{2} \cos (k)}}
\end{gathered}
$$

where $0 \leq k_{c 1} \leq \pi$ is the solution of the equation $\varepsilon_{k, 1}\left(k_{c 1}\right)=0$, and $0 \leq k_{c 2} \leq \pi$ is determined from the condition $\varepsilon_{k, 2}\left(k_{c 2}\right)=0$. This situation happens if both $\varepsilon_{k, 1,2}$ have extremal values as a function of $k$ only for $k=0, \pm \pi$. It turns out that $\gamma_{1,2}$ are nonzero only when the difference between the populations of the Fermi-Dirac seas, connected with upper and lower bands, exists.

\subsection{Analysis of the spectral features}

Equations (8) imply several different possibilities of the ground state behavior of the studied model. We know that the mean-field approximation often does not provide correct values of critical constants (critical values of exchange integrals or magnetic field, at which quantum phase transitions take place), this is why, we limit ourselves in what follows mostly with the qualitative mean field analysis of the situation in the considered model in the ground state. In what follows we shall consider the properties of the bands Eqs. (8), (9). In such analysis, as usually for one-dimensional systems, the edges of the bands produce van Hove singularities, which manifest themselves in quantum phase transitions. They are governed, e.g., by the magnetic field, which regulates the filling of the bands.

The case without dimerization, i.e. with $\mu_{1}=\mu_{2}$ and $J_{1}=J_{2}, J_{1}^{z}=J_{2}^{z}$, at $H=0$ was considered, e.g., in Refs. 17,18 . This case in the magnetic field was studied in Refs. 19. The isotropic dimerized case at $H=0$ for $J_{N N}=J_{N N}^{z}=0$ was studied in the antiferromagnetic case in Refs. 20,21. On the other hand, in the absence of the magnetic field, $H=0$, the magnetically isotropic case permits the exact solution for the ground state $[22,23]$ for special choices of exchange constants $J_{1}, J_{2}$ and $J_{N N}$.

If $\mu_{1}=\mu_{2}=\mu$ and $J_{N N}=J_{N N}^{z}=0$ (i.e. in the absence of the NNN interactions), one has $A=0$, and, therefore, $m_{1,2}^{z}=m^{z}=(1 / 2)-\left(k_{c 1}+k_{c 2}\right) / 2 \pi$, and $\gamma_{N N 1,2}=\gamma_{N N}=$ $=(1 / 2 \pi)\left[\sin \left(k_{c 1}\right)+\sin \left(k_{c 2}\right)\right]$. In this case the upper band has one maximum at $k=0$ and two minima at $k= \pm \pi$, while the lower band has one minimum at $k=0$ and two maxima at $k= \pm \pi$. The gap between bands is caused by the difference between $J_{1}$ and $J_{2}$. Both bands are totally filled in the ground state (i.e. $k_{c 1,2}=\pi$, hence $\gamma_{1,2}=$, $=\gamma_{N N}=0, m^{z}=-1 / 2$ ) for

$$
H<H_{s 1}=(1 / \mu)\left[-\left|J_{1}+J_{2}\right|+\left(J_{1}^{z}+J_{2}^{z}\right) / 2\right] .
$$

In this spin-saturated phase all excitations are gapped. The low-temperature magnetic susceptibility and specific heat are exponentially small for a gapped phase. Both bands are empty in the ground state (i.e. $k_{c 1,2}=0$, thus $\gamma_{1,2}=$ $\left.=\gamma_{N N}=0, m^{z}=1 / 2\right)$ for $H>H_{s 2}=(1 / \mu)\left[\left|J_{1}+J_{2}\right|-\right.$ $\left.-\left(J_{1}^{z}+J_{2}^{z}\right) / 2\right]$. In this spin-saturated phase all excitations are also gapped.

If the lower band is totally filled and the upper band is empty in the ground state (i.e. $k_{c 1}=0, k_{c 2}=\pi$, hence $m^{z}=0, \gamma_{N N}=0$ ) excitations are also gapped (except of the case, when $\left.H=H_{c}=(1 / \mu)\left|B_{1}-B_{2}\right|\right)$. In all other cases either upper band, or lower one, is partly filled in the ground state, with gapless excitations (holes in the Fermi-Dirac sea, related to the corresponding partly filled band). Gapless excitations imply finite low-temperature magnetic susceptibility, for $m^{z}=0$, or divergent low- $T$ magnetic susceptibility for $m^{z} \neq 0$, and the low-temperature specific heat proportional to $T$. If $\left(J_{1}^{z}+J_{2}^{z}\right)>0$ and $m^{z} \geq 0$ (or $\left(J_{1}^{z}+J_{2}^{z}\right)<0$ and $m^{z} \leq 0$ ), then the energies of states in the upper band are always positive (i.e. $k_{c 1}=0$ ), and, hence, the upper band is empty in the ground state. The lower band, depending on $H$, can be totally filled (i.e. $k_{c 2}=\pi$, therefore, $m^{z}=0$, $\gamma_{N N}=0$ ), empty (i.e. $k_{c 2}=0$, therefore, $m^{z}=1 / 2$, $\gamma_{N N}=0, \gamma_{1,2}=0$ ), and partly filled in the ground state. The lower band is totally filled for $H<H_{c}$ at $T=0$. In this phase all excitations are gapped, with exponentially small low-temperature magnetic susceptibility and specific heat.

The lower band is empty at $T=0$ for $H>H_{S}=$ $=(1 / \mu)\left[\left|J_{1}+J_{2}\right| \mp\left(J_{1}^{Z}+J_{2}^{z}\right) / 2\right]$, where the upper sign corresponds to the case with $m^{z}>0$, and the lower one is related to the situation with $m^{z}<0$. Obviously, this phase exists if $\left|J_{1}+J_{2}\right|> \pm\left(J_{1}^{z}+J_{2}^{z}\right) / 2$. In this spin-saturated phase all excitations are gapped, too. For $H_{c}<H<H_{s}$ low-energy excitations (holes in the Dirac sea, related to the lower band) are gapless, and the low-temperature specific heat is proportional to $T$, while the low- $T$ magnetic susceptibility is finite for $m^{z}=0$ and divergent for $m^{z} \neq 0$. At $H_{C}$ and $H_{S}$ second order quantum phase transitions take place. If $\left(J_{1}^{z}+J_{2}^{z}\right) \leq 0$ and $m^{z} \geq 0$ (or $\left(J_{1}^{z}+J_{2}^{z}\right) \geq 0$ and $\left.m^{z} \leq 0\right)$, then energies of states in the upper band can be negative (i.e. $k_{c 1} \geq 0$ ). In this case up to four second order quantum phase transitions can take place. For $H<H_{s 1}$ the system is in the spin-polarized phase with $m^{z}=-1 / 2$. For $H_{s 1} \leq H \leq H_{c 1}=(1 / \mu) \quad\left[-m^{z}\left(J_{1}^{z}+J_{2}^{z}\right)-\left|B_{1}-B_{2}\right|\right]$ (for $\left.-m^{z}\left(J_{1}^{z}+J_{2}^{z}\right)>\left|B_{1}-B_{2}\right|\right)$ and for $H_{c 2}=(1 / \mu) \times$ $\times\left[-m^{z}\left(J_{1}^{z}+J_{2}^{z}\right)+\left|B_{1}-B_{2}\right| \leq H \leq H_{s 2}\right]$ the system is in the gapless phases. For $H_{c 1} \leq H \leq H_{c 2}$ the system is in the gapped phase with empty upper band and totally filled lower band, where $m^{z}=0$. Finally, for $H>H_{s 2}$ the model is in the spin-polarized phase with $m^{z}=1 / 2$. 
The general situation with $J_{N N} \neq 0, J_{N N}^{z} \neq 0$, is more complicated, and, therefore, more interesting.

(i) This case takes place when both upper and lower bands Eq. (8) empty in the ground state, i.e. $k_{c 1}=k_{c 2}=0$. This case corresponds to the spin-saturated (ferromagnetic) phase, with $m_{1}^{z}=m_{2}^{z}=1 / 2, \gamma_{1,2}=\gamma_{N N 1,2}=0$. It happens even in the absence of the external magnetic field for the case $J_{1}^{z}+J_{2}^{z}-2 J_{N N}^{z} \pm 2 J_{N N}>\left|J_{1} \pm J_{2}\right|$, for which values of the exchange constants the spontaneous ferromagnetic order takes place in the ground state of the model. For that situation, obviously, the line $H=0$ is the line of the first order quantum phase transition (the magnetic field removes the two-fold degeneracy of the ferromagnetic ground state). If the above mentioned condition for exchange constants is not fulfilled, i.e. there is no spontaneous ferromagnetism in the model, the case with totally empty Fermi-Dirac seas can exist for large enough values of the external magnetic field. Here, for $H \neq 0$, the spin-saturated ground state situation is related to $H>H_{s}$, the critical field of the second order quantum phase transition to the spin-saturated phase, which is determined by one of the values of

$$
\begin{gathered}
H_{s 1,2}=\frac{1}{4 \mu_{1} \mu_{2}} \mid-\left(\mu_{1}+\mu_{2}\right)\left(J_{1}^{z}+J_{2}^{z}-2 J_{N N}^{z}+2 J_{N N}\right) \pm \\
\pm\left[\left(\mu_{1}-\mu_{2}\right)^{2}\left(J_{1}^{z}+J_{2}^{z}-2 J_{N N}^{z}+2 J_{N N}\right)^{2}+\right. \\
\left.\quad+4 \mu_{1} \mu_{2}\left(J_{1}+J_{2}\right)^{2}\right]^{1 / 2} \mid
\end{gathered}
$$

For $H>H_{s}$ excitations, related to both bands, are gapped, which reveals itself, e.g., in exponentially small values of the low-temperature magnetic susceptibility and specific heat.

(ii) The low-energy band can be totally filled, and the upper in energy band can be empty at $T=0$, i.e. $k_{c 1}=0$, $k_{c 2}=\pi$, like in Figs. 2 and 4. Then it is seen from Eqs. (17) that one has $\gamma_{N N 1}=-\gamma_{N N 2}$. One can also see that the $z$-projection of the total spin of the model is zero in this phase, $m_{1}^{z}+m_{2}^{z}=0$ (but not the $z$-projection of the total magnetization, which can be nonzero in the general case $\mu_{1} \neq \mu_{2}$ ). This situation is characterized by the gap for the excitations, related to the upper band, and also can be characterized by the gap for the lower-band excitations (holes in the Dirac sea), which exists for $\varepsilon_{k, 2}<0$ for all $k$. This lower-band gap can be closed for some values of parameters of the Hamiltonian. For example, it can be related to the critical value of the field, $H=H_{c}$, at which the second order quantum phase transition to the phase with lower-band gapless excitations takes place. For $H>H_{c}$ the excitations, related to the lower band, become gapless, and, therefore, the low-temperature magnetic susceptibility is finite, and the low-temperature specific heat becomes linear in $T$. On the other hand, the lower band can be totally filled at $T=0$, but excitations, related to this band (holes in the Dirac sea) can be gapless (in this case $H_{c}=0$ ). Here the low-temperature magnetic susceptibility of the model is finite, and the low-temperature specific heat is proportional to $\sqrt{T}$. Further growth of $H$ in the ground state can produce the second order quantum phase transition to the spin-saturated phase for $H=H_{S}$, which corresponds to the situation, at which the lower band becomes totally empty, and excitations, related to it, become gapped. In this case for $\mu_{1}=\mu_{2}=\mu$, we have $A_{1}=2 \mu H$. In the case of small NNN interactions, we get $A \approx 0, m_{1,2}^{z}=\gamma_{N N, 1,2} \approx 0$. The parameters $\gamma_{1,2}$ are obtained as the solution of equations

$$
\begin{aligned}
& \gamma_{1}+\alpha \gamma_{2}=-\frac{1}{2 \pi}(1+\alpha) E(x), \\
& \gamma_{1}-\alpha \gamma_{2}=-\frac{1}{2 \pi}(1-\alpha) K(x),
\end{aligned}
$$

where $\alpha=B_{2} / B_{1}$,

$$
x=\frac{4 \alpha}{(1+\alpha)^{2}}
$$

and $K(x)$ and $E(x)$ are the complete elliptic integrals of the first and second kinds, respectively. $2 \mu H_{c}$ is determined by the value of the gap between bands. The gap, in turn, is determined by the value

$$
\left|B_{1}-B_{2}\right|=\left|\left(J_{1}-J_{2}\right)-2\left(J_{1}^{z} \gamma_{1}-J_{2}^{z} \gamma_{2}\right)-4 J_{N N}\left(\gamma_{1}-\gamma_{2}\right)\right| \text {. }
$$

The solution for the case with gapped excitations corresponds to $\gamma_{1} \neq \gamma_{2}$, existing even for $J_{1}=J_{2}, J_{1}^{z}=J_{2}^{z}$. For small values of $J_{N N}$ one has

$$
\alpha \approx \frac{J_{2}-2 J_{1}^{z} \gamma_{2}}{J_{1}-2 J_{1}^{z} \gamma_{1}}\left(1-4 J_{N N}\left[\frac{\gamma_{1}}{J_{2}-2 J_{2}^{z} \gamma_{2}}-\frac{\gamma_{2}}{J_{1}-2 J_{1}^{z} \gamma_{1}}\right]\right) \text {. }
$$

(iii) The lower band can be partly filled, while the upper band is empty in the ground state, i.e. $k_{c 1}=0$, like in Fig. 3 . This case is characterized by the nonzero value of the $z$-projection of the total spin of the model per cell (different, generally speaking, from the nominal value, $1 / 2$ ), $m_{1}^{z}+m_{2}^{z}=1-k_{c 2} / \pi$, which can be spontaneous, i.e. it can exist even for $H=0$. Low-energy excitations (holes in the lower band) are gapless, and, therefore, the low- $T$ specific heat is proportional to the temperature. For nonzero spontaneous spin moment the low-temperature magnetic susceptibility is divergent, while if the moment is caused by the nonzero value of the field $H$, the low- $T$ susceptibility is finite. At $H=0$ in the ground state for the case with nonzero spontaneous magnetization the first order quantum phase transition takes place, because the magnetic field removes the degeneracy of the ground state. The growth of the magnetic field yields a second order quantum phase 
transition at $H=H_{S}$ to the spin-polarized state with the nominal value of $M^{z}$ with only gapped excitations. In the case of small $k_{c 2}$ one has the solution of self-consistency equations $A \approx 0, m_{1,2}^{z} \approx(1 / 2)-\left(k_{c 2} / 2 \pi\right)$ (equal to the ground state spontaneous magnetization), $\gamma_{N N, 1,2} \approx$ $\approx k_{c 2} / 2 \pi$, and $\gamma_{1,2} \approx-\left(k_{c 2} / 4 \pi\right)$. Here the critical value of $k_{c 2}$ is determined at $H=0$ by

$$
\frac{k_{c 2}}{2 \pi} \approx \frac{J_{1}^{z}+J_{2}^{z}-2 J_{N N}^{z}+2 J_{N N}+J_{1}+J_{2}}{3\left(J_{1}^{z}+J_{2}^{z}\right)-8 J_{N N}^{z}-4 J_{N N}},
$$

or

$$
\frac{k_{c 2}}{2 \pi} \approx \frac{J_{1}^{z}+J_{2}^{z}+2 J_{N N}^{z}-2 J_{N N}-J_{1}-J_{2}}{J_{1}^{z}+J_{2}^{z}-8 J_{N N}^{z}-12 J_{N N}},
$$

for $\left(J_{1}+J_{2}\right)$, being negative or positive, respectively. Such solutions must exist only for small values of $k_{c 2}$.

(iv) At $T=0$ the lower band can be totally filled, i.e. $k_{c 1}=\pi$, while the upper band is partly filled. In this case one has also, as in the previous case, possibility of the nonzero value of the $z$-projection of the total spin of the model, spontaneous (generally speaking different from $1 / 2$ ), or caused by the external magnetic field. Depending on that fact (i.e., whether the moment is spontaneous, or not), the low-temperature magnetic susceptibility is divergent, or finite. Again, lowest in energy excitations (holes in the Fermi-Dirac sea, connected with the upper band) are gapless, and the low-temperature specific heat is proportional to $T$. Also, at $H>H_{S}$ the model appears in the spin-polarized ground state, as in the previous case. In the situation with nonzero spontaneous magnetization in the ground state, the first order phase transition takes place, because the field removes the degeneracy of the ground state. However, this situation is different from the previous one, because, if the bottom of the upper band is lower than the top of the lower band, at $H_{c 1}, H_{c 2}<H_{s}$ two quantum second order phase transitions take place, with the square root feature in the behavior of the magnetic susceptibility. It turns out that these phase transitions are between phases with gapless excitations. On the other hand, if the bottom of the upper band is higher than the top of the lower band, one has two second order quantum phase transitions, and, hence, two critical values of the magnetic field $H_{c 1}$ and $H_{c 2}$ with the values smaller than $H_{s}$. Between those field values there are no magnetic eigenstates of the Hamiltonian, and, therefore, the field dependence of the magnetization has to manifest a magnetic plateau for $H_{c 1}<H<H_{c 2}$.

(v) Then, both, lower and upper bands can be partly filled in the ground state. The properties of this situation are similar to the ones of the previous case with two critical values of the field, $H_{c}$ and $H_{s}$. The transition at $H_{c}$ is between phases with gapless excitations. At $H=0$ one has to observe a first order phase transition, because the field removes the degeneracy of the ground state. Cases (iv) and (v) are related to large values of the NNN interactions.

(vi) Finally, the case with both upper and lower band filled (i.e. $k_{c 1}=k_{c 2}=\pi$ ) at $T=0$ formally corresponds to the solution of self-consistency equations with $m_{1}^{z}=$ $=m_{2}^{z}=-1 / 2, \gamma_{1,2}=\gamma_{N N 1,2}=0$.

The situation becomes more complicated for the case, where $\varepsilon_{k, 1,2}$ as functions of $k$ have additional, with respect to $k=0, \pm \pi$, extremum values. Such situation corresponds to more than one Fermi-Dirac sea per band, which are situated symmetrically with respect to $k=0$. Obviously, it exists only for nonzero $J_{N N}$ and $J_{N N}^{z}$. It implies that in Eqs. (17) one has to replace integration from $-k_{c 1,2}$ to $k_{c 1,2}$ to the integration over the intervals, where $\varepsilon_{k, 1,2}<0$. Here the system can be in incommensurate structures in the ground state (it is in the incommensurate phase if the lowest value of the energy corresponds to some $k \neq 0, \pm \pi$ ). For the cases (i) and (vi) there are no incommensurate structures, obviously. For (ii) additional extremum values of only lower branch matter, see Fig. 4. In the gapped case (or in the gapless one, if $H_{c}=0$ ), additional field-governed second order phase transition between commensurate and possible incommensurate gapless phases takes place for $T=0$, cf. Fig. 4. For (iii), as in the previous case, additional extremum values of only lower branch matter. Again, additional field-governed second order quantum phase transition between commensurate and possible incommensurate gapless phases takes place, see Fig. 5. In the cases (iv) and (v) additional extremum values of both, upper and lower bands matter, see Fig. 6. In the case (iv), with a magnetic plateau, in addition to $H_{c 1,2}$ two more quantum phase transitions can take place. Both of those second order quantum phase transitions are between commensurate and possible incommensurate phases with gapless excitations. For (iv) with the bottom of the upper

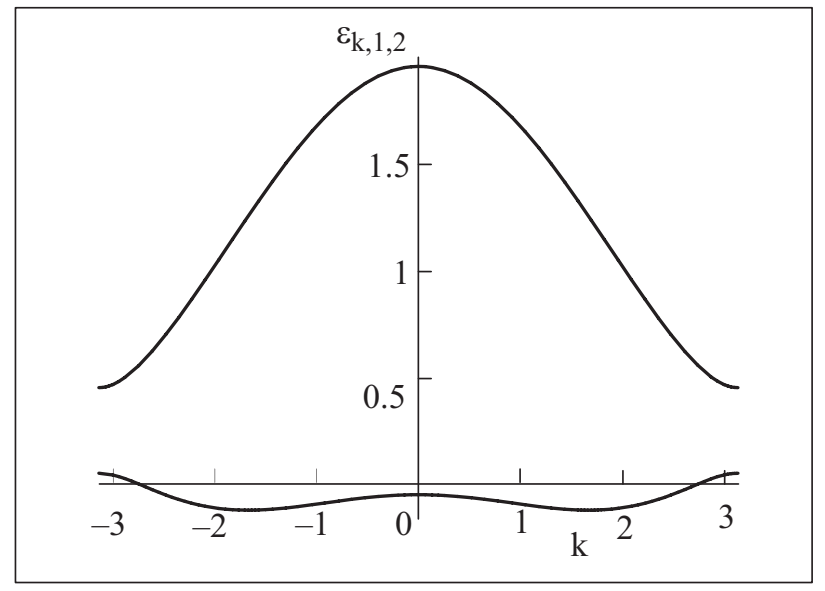

Fig. 5. Dispersion laws $\varepsilon_{k, 1,2}$ of the studied model with empty upper band and partly filled lower band. 


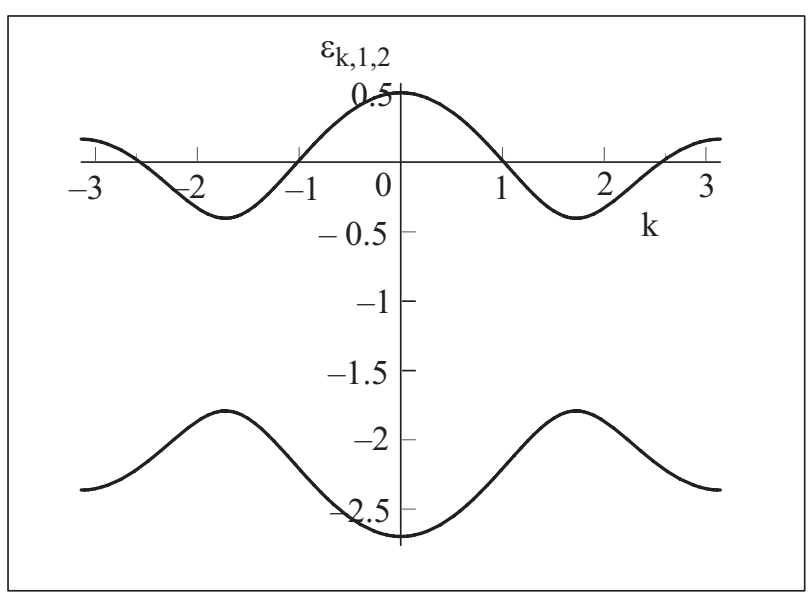

Fig. 6. Dispersion laws $\varepsilon_{k, 1,2}$ of the studied model with partly filled upper band with two minima and totally filled lower band with two minima.

band being higher than the top of the lower band, as well as for (v), again, two additional ground state phase transitions can take place, also between commensurate and possible incommensurate phases with gapless excitations.

Notice that low-energy excitations correspond to the states with positive energies, i.e. the dispersion laws for those excitations are related to $\varepsilon_{k, 1,2}$ for branches Eqs. (8) with positive energies and to $\left|\varepsilon_{k, 1,2}\right|$ for holes for the branches with negative energies.

It is interesting to observe that proposed recently exactly solvable model of a spin- $1 / 2$ chain with alternating $\mathrm{NN}$ interactions and multiple spin exchange [24] manifest properties, similar to the ones of the present model.

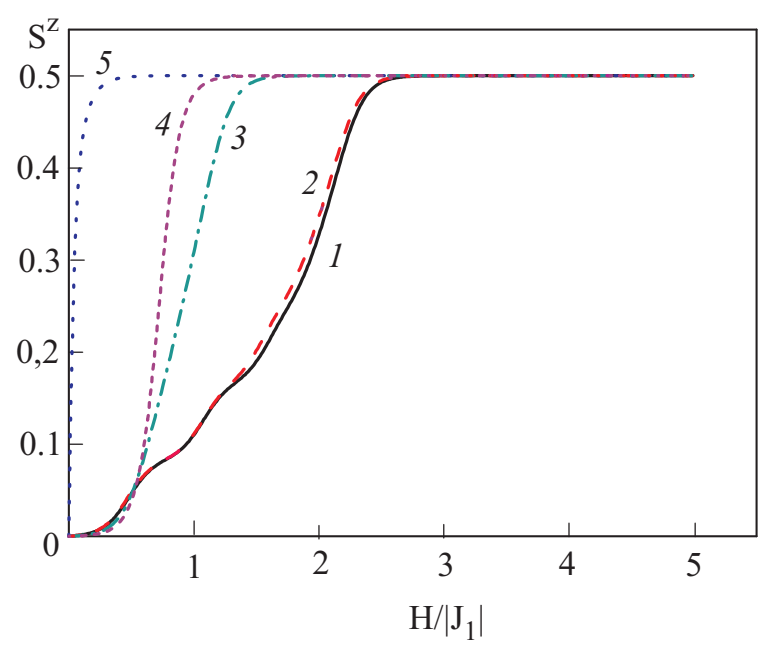

Fig. 7. The magnetization per site of the considered model for $J_{N N}= \pm 0.1\left|J_{1}\right|$ as a function of $H$ at $T=0.1\left|J_{1}\right|$. Notations here and in Figs. $7-14$ are as follows. $J_{1}=1, J_{2}=1.2, J_{N N}=0.1\left|J_{1}\right|$ (1); $\quad J_{1}=1, \quad J_{2}=1.2, \quad J_{N N}=-0.1\left|J_{1}\right| \quad(2) ; \quad J_{1}=1, \quad J_{2}=-1.2$, $J_{N N}=-0.1\left|J_{1}\right|(3) ; J_{1}=1, J_{2}=-1.2, J_{N N}=0.1\left|J_{1}\right|(4) ; J_{1}=-1$, $J_{2}=1.2, J_{N N}=0.1\left|J_{1}\right|(5)$.

\section{Exact diagonalization: thermodynamics}

Now let us check the features of the behavior of the spin-1/2 chain with alternating NN and (homogeneous) NNN interactions, obtained with our mean field analysis with the results of exact diagonalization of small clusters (short chains). Most of our calculations were performed for the chains containing 14 spins (even numbers). The number of spins in the chain was limited by the exponential growth of the computation time, however the accuracy of our calculations was sufficient to reproduce the features of the magnetization, magnetic susceptibility and specific heat of the considered model down to temperatures of order of 0.01 of the smallest absolute value of the $\mathrm{NN}$ exchange coupling. In our calculations we used arbitrary units with Boltzmann's constant, Bohr's magneton and $g$-factor being equal to unity (we studied the case of $\mu_{1}=\mu_{2}=\mu$ ). Figs. 7-15 present results of those calculations for $S^{z} \equiv M^{z}(H) / \mu$ at $T=0.1\left|J_{1}\right|, \chi^{z}(T)$ and $c(T)$ for $H=0$ for the model with magnetically isotropic $\mathrm{NN}\left(J_{1,2}=J_{1,2}^{z}\right)$ and $\mathrm{NNN}$ $\left(J_{N N}=J_{N N}^{z}\right)$ interactions for $J_{1}= \pm 1, J_{2}= \pm 1.2, J_{N N}=$ $= \pm 0.1, \pm 0.3, \pm 0.8$ (except of the trivial case with all exchange constants being ferromagnetic).

One can see that for the cases with antiferromagnetic $\mathrm{NN}$ interactions for any sign of NNN interactions, and for alternating in signs NN interactions with antiferromagnetic NNN interactions low-lying excitations are gapped, and, therefore, the magnetization is small for small values of the field (cf. Figs. 7, 10, and 13), and the magnetic susceptibility (cf. Figs. 8, 11, and 14) and specific heat (cf. Figs. 9, 12, and 15) are exponentially small at low temperatures. The same is true for larger values of NNN exchange constants for the case with ferromagnetic $\mathrm{NN}$ and antiferromagnetic NNN interactions, and for alternating in signs $\mathrm{NN}$ interactions with ferromagnetic NNN couplings. It implies that the mean field like approximation correctly (at least in these cases) reproduces

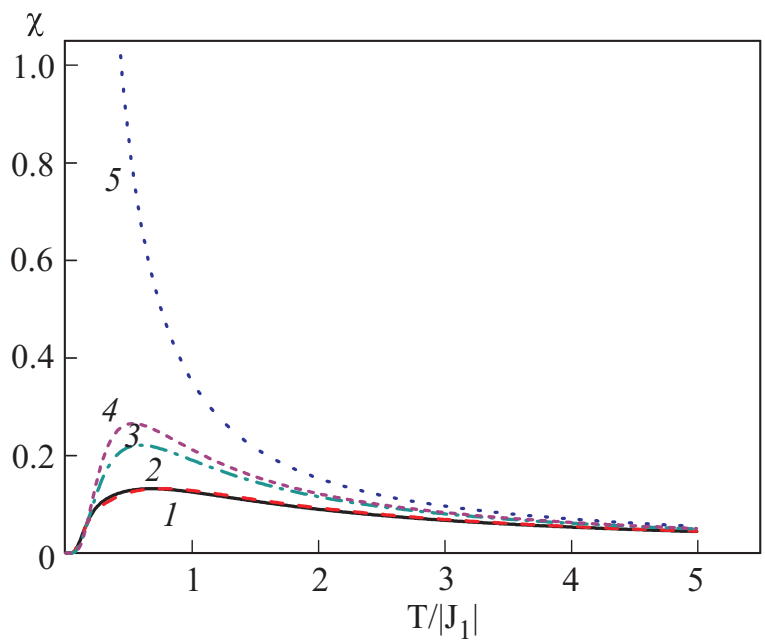

Fig. 8. The magnetic susceptibility per site of the considered model for $J_{N N}= \pm 0.1\left|J_{1}\right|$ as a function of $T$ at $H=0$. 


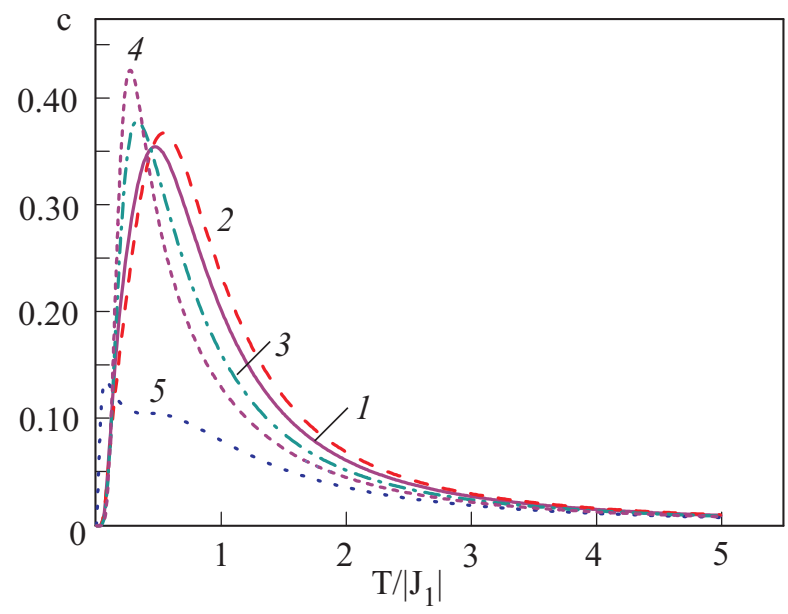

Fig. 9. The specific heat per site of the considered model for $J_{N N}= \pm 0.1\left|J_{1}\right|$ as a function of $T$ at $H=0$.

such a behavior, cf. (ii), like in Fig. 2, or (ii) with possible additional Fermi-Dirac seas of the lower band, see Fig. 4. The large region of an exponentially small magnetization implies a large value of the spin gap.

On the other hand, for the case with alternating in signs NN interaction for $J_{N N}=0.1\left|J_{1}\right|$, and for ferromagnetic NN interactions for $J_{N N}=-0.1\left|J_{1}\right|,-0.3\left|J_{1}\right|$ we see the divergency of the low-temperature magnetic susceptibility, see Figs. 8 and 11 (which manifests the spontaneous magnetization in the ground state), additional low-temperature narrow maxima for the specific heat, see Figs. 9 and 12, and a metamagnetic-like transition at small values of the field of the low-temperature magnetization, see Figs. 7, 10, and 13. This situation is, probably, related to the cases (i) or (iii), cf. Fig. 3, with one Fermi-Dirac sea, or with possible additional Fermi-Dirac seas of the lower band in the mean field like analysis. Notice that in those cases the bandwidth of the lower band is narrow, which produces the metamagnetic-like transition to the spin-polarized phase. Our exact diagonalization re-

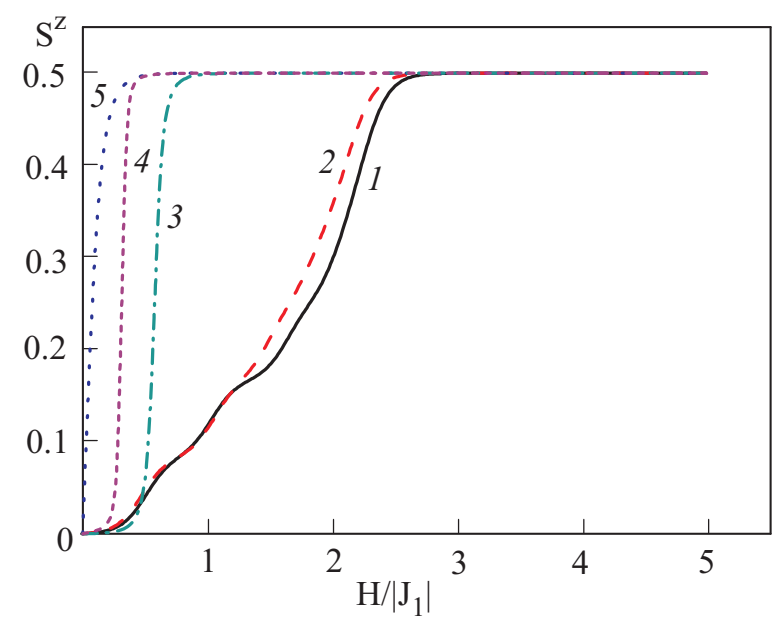

Fig. 10. The same as in Fig. 7, but for $J_{N N}= \pm 0.3\left|J_{1}\right|$.

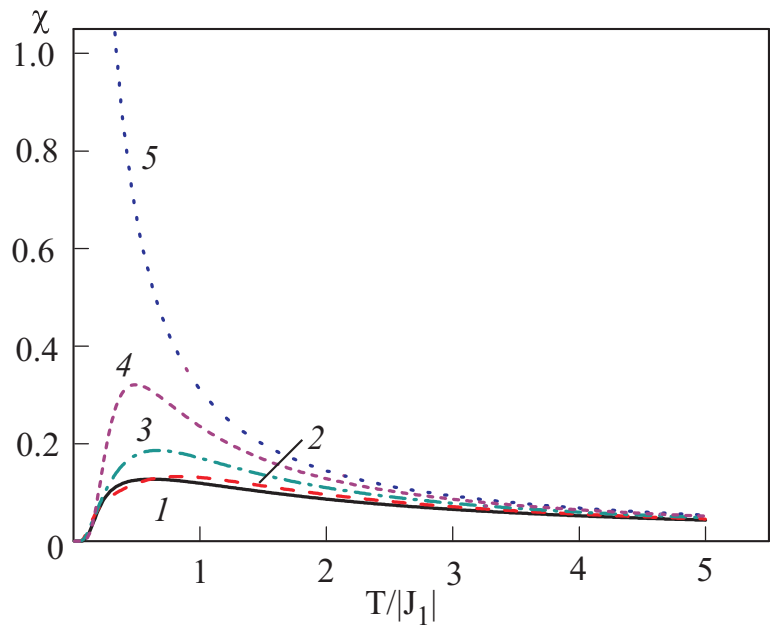

Fig. 11. The same as in Fig. 8, but for $J_{N N}= \pm 0.3\left|J_{1}\right|$.

sults imply that at least for the calculated cases the situations like in (iv), (v) and (vi) are not realized in the studied spin chain (zig-zag ladder).

In order to get more information about quantum phase transitions with respect to external field, we calculated the lowest energy levels of finite chain fragments formed up to 16 spins for each value of total spin of clusters for the isotropic case $\left|J_{1,2}^{z}\right|=J_{1,2},\left|J_{N N}^{z}\right|=J_{N N}$. For this purpose we used branching diagram technique and the Davidson's method (the modification of the Lanczos scheme for the determination of lowest eigenvalues of large matrices). We found the jumps of the ground state total spin $S$ due to change of coupling parameter $J_{N N}$ in rather narrow region. This gives us the idea to find simple approximate estimate for critical value of this parameter, which corresponds to the beginning of the destruction of ferromagnetic ground state of the chain, by means of study the stability of ferromagnetic state with respect to the inversion of a single site spin. It is easily shown, that the energy spectrum of the states with one inverted spin is defined by formula

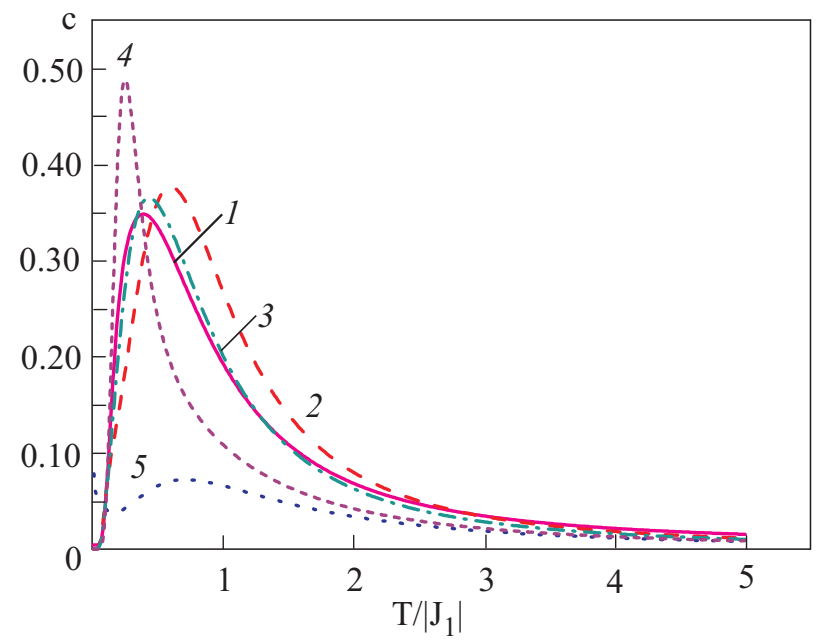

Fig. 12. The same as in Fig. 9, but for $J_{N N}= \pm 0.3\left|J_{1}\right|$. 


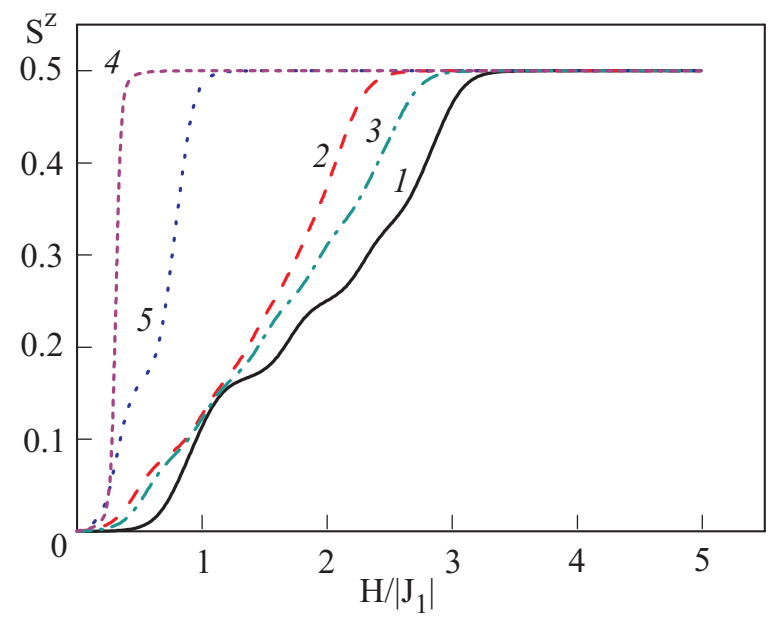

Fig. 13. The same as in Fig. 7, but for $J_{N N}= \pm 0.8\left|J_{1}\right|$.

$$
\begin{gathered}
\delta \equiv E(k)-E_{f}=-\frac{1}{2}\left(J_{1}+J_{2}+2 J_{N N}[1-\cos (k)]+\right. \\
\left.+\sqrt{J_{1}^{2}+J_{2}^{2}+2 J_{1} J_{2} \cos (k)}\right),
\end{gathered}
$$

where $E_{f}$ is the energy of the ferromagnetic (totally spin-polarized) state, and $E(k)$ is the energy of the single inverted spin with respect to the spin-polarized state. Let $J_{1,2}<0$ and $J_{N N}>0$. In this case for the infinite chain it can be shown that the ferromagnetic state is unstable (non-positive values of $\delta$ ), if the coupling $J_{N N}$ takes values from the interval

$$
\frac{J_{1} J_{2}}{2\left|J_{1}-J_{2}\right|} \geq J_{N N} \geq \frac{J_{1} J_{2}}{2\left|J_{1}+J_{2}\right|} .
$$

In other words, the expression

$$
J_{N N}=\frac{J_{1} J_{2}}{2\left|J_{1}+J_{2}\right|}
$$

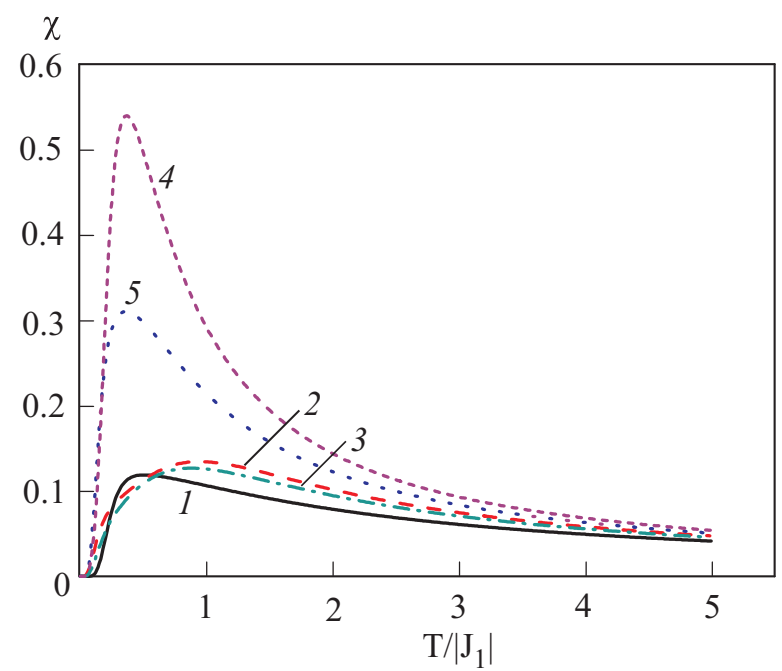

Fig. 14. The same as in Fig. 8, but for $J_{N N}= \pm 0.8\left|J_{1}\right|$.

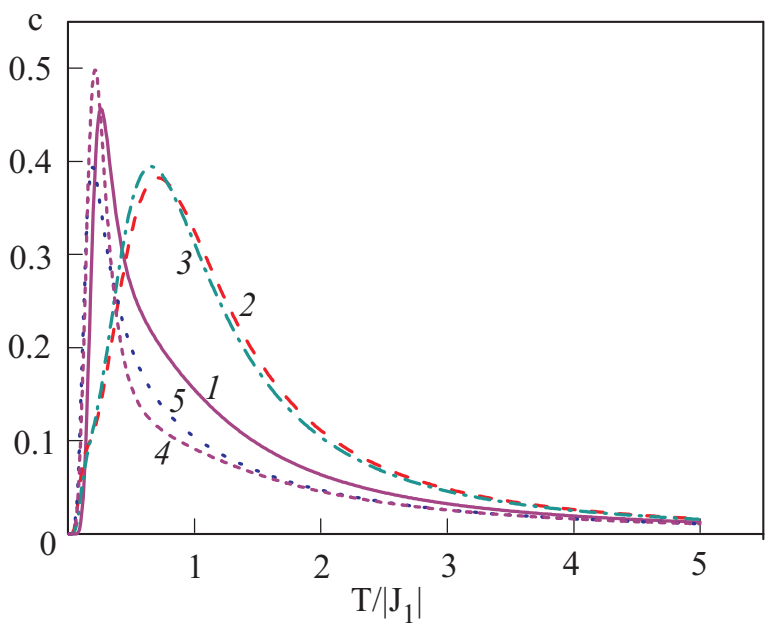

Fig. 15. The same as in Fig. 9, but for $J_{N N}= \pm 0.8\left|J_{1}\right|$.

may be a good estimate the for critical value of $J_{N N}$. Our numerical calculations for linear chain fragments support this conclusion. Our calculations, compare with the results of Ref. 22, for chain fragments with periodic boundary conditions (Fig. 16) demonstrate that this estimate corresponds to the exact degeneracy of the ferromagnetic and the lowest singlet states regardless on the size of fragment.

Simultaneously, all the lowest states from other subspaces with the given total spin have larger energy in the vicinity of this point. It means that Eq. (27) determines the critical surface in the space of three coupling parameters, that corresponds to the first order transition between the ferromagnetic and the singlet states.

On the other hand, we see that for antiferromagnetic NN interactions additional field-induced ground state phase transitions can take place. For example, Figs. 7, 10, and 13 manifest features of the behavior at the values of

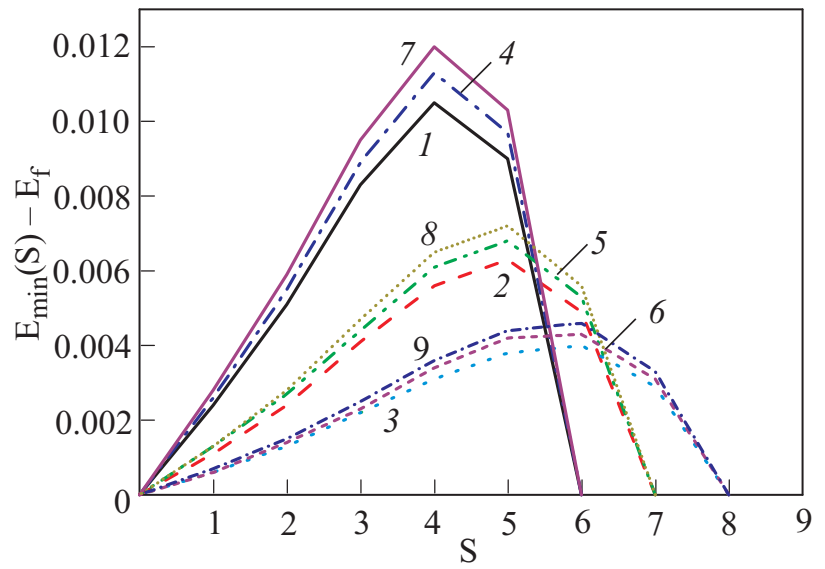

Fig. 16. Lowest energies with the specified value of the total spin $S$ of spin-1/2 chain fragments consisting of $N=12-16$ spins with $J_{1}=-1$ for the cases: $J_{2}=-1, J_{N N}=0.25, N=12(1), N=14(2)$, $N=16(3) ; J_{2}=-1.2, J_{N N}=3 / 11, N=12(4), N=14(5), N=16$ (6); $J_{2}=-1.5, J_{N N}=0.3, N=12(7), N=14(8), N=16(9)$. 


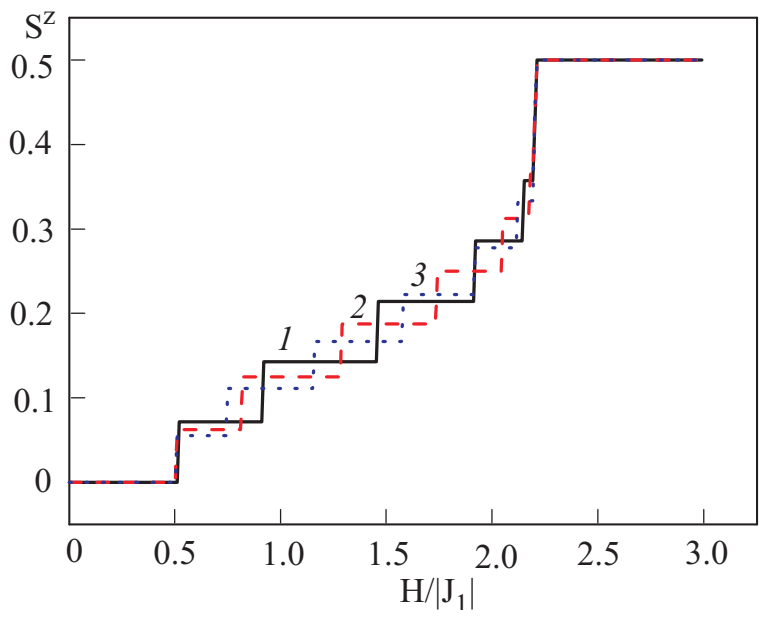

Fig. 17. The magnetization of the chain consisting of 14 (1), $16(2)$, and 18 spins $1 / 2$ (3) with antiferromagnetic alternating NN interactions and antiferromagnetic NNN couplings as a function of the magnetic field at $T=0$ calculated using Davidson's method.

the magnetization per spin equal to approximately 0.08 , $0.16,0.25$, and/or 0.34 . We performed calculations for $J_{N N}= \pm 2.5\left|J_{1}\right|$ (not shown here), and they also reveal additional features, clearly demonstrating magnetic plateaux at approximately 0.16 of the magnetization per spin. Those features in the behavior of the magnetization are followed, naturally, by the features in the magnetic field behavior of the magnetic susceptibility and specific heat at low temperatures at the same values of the magnetic field, at which features of the magnetization take place. It is not clear, however, whether all those additional field-induced features are related to real quantum phase transitions, or they are connected with the finite-size effects. At least, most of such a features are reproduced in all performed calculations (up to 18 spins). Obviously, additional plateaux in the magnetization behavior imply additional bands of low lying excitations of

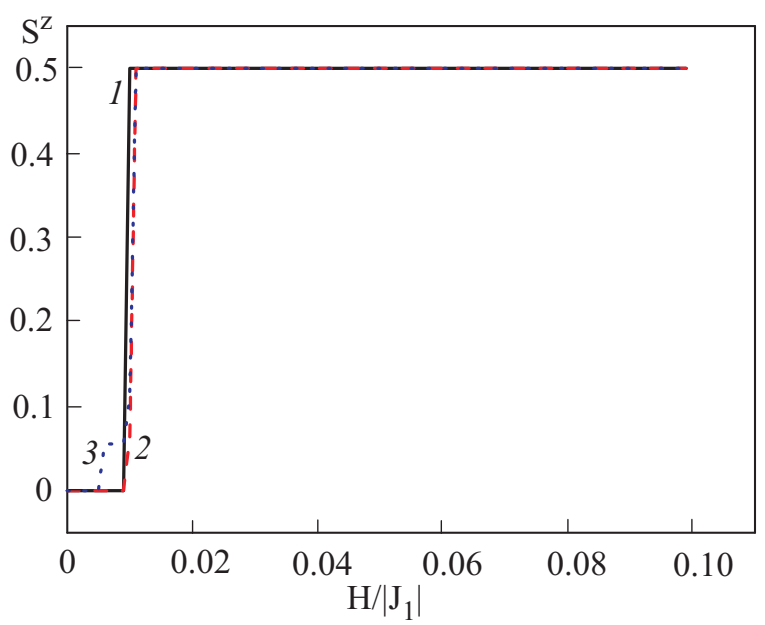

Fig. 18. The same as in Fig. 17, but for the chain with ferromagnetic alternating $\mathrm{NN}$ and antiferromagnetic $\mathrm{NNN}$ interactions.

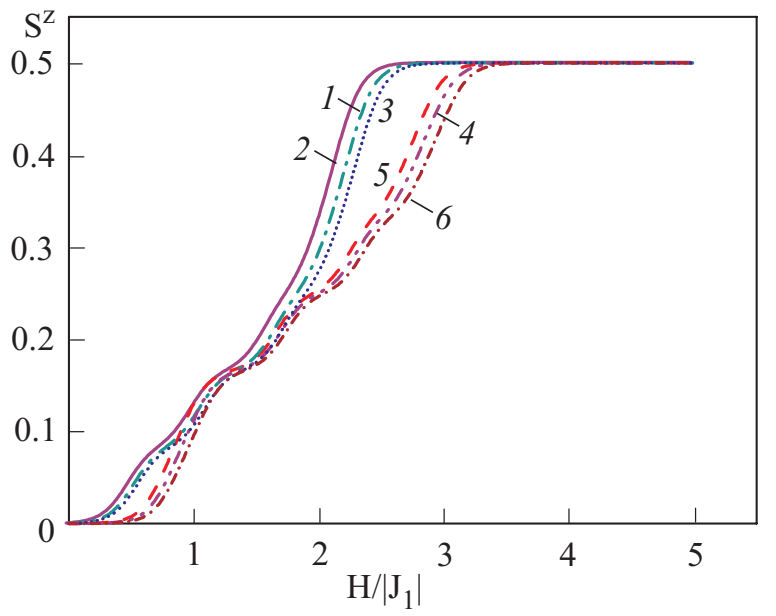

Fig. 19. The magnetization of the spin-1/2 chain with antiferromagnetic alternating $\mathrm{NN}\left(J_{1}=1, J_{2}=1.2\right)$ interactions and antiferromagnetic NNN couplings as a function of the magnetic field at low temperatures for the isotropic case, and for the weak easy-axis and easy-plane magnetic anisotropy. The notations are as follows. For $J_{N N}=0.3$ the isotropic case (1); the easy-axis case (2); the easy-plane case (3). For $J_{N N}=0.8$ the isotropic case (4); easy-axis case (5); the easy-plane case (6).

the considered chain, and, therefore, our mean field like analysis, based on two bands for magnetic excitations, is, probably, limited to relatively small values of NNN interactions.

We also performed calculations of the ground state magnetization of chains, containing 14, 16, and 18 spins using the Davidson's method. The results of calculations with periodic boundary conditions for the cases with $J_{N N}=0.3$ with $J_{1}=1, J_{2}=1.2$, and $J_{1}=-1, J_{2}=-1.2$, are given in Figs. 17 and 18, respectively. One can see the qualitative agreement between these calculations and the ones, using the exact diagonalization scheme.

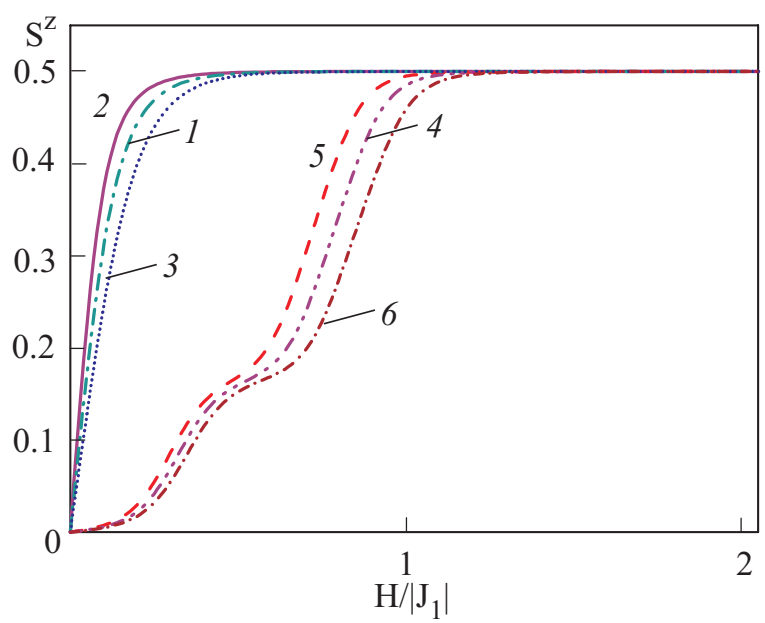

Fig. 20. The same as in Fig. 19 (with the same notations), but for the spin chain with ferromagnetic alternating $\mathrm{NN}$ interactions $\left(J_{1}=-1, J_{2}=-1.2\right)$. 


\section{Magnetic anisotropy}

Figures 19 and 20 show the behavior of the low-temperature $\left(T=0.1 \mid J_{1}\right)$ magnetization as a function of $H$ for the model with $J_{1}=1, J_{2}=1.2$ and $J_{1}=-1, J_{2}=-1.2$, respectively, for $J_{N N}=0.3,0.8$, for the case of a weak uniaxial magnetic anisotropy, the axis of which is directed along the magnetic field (the data are presented for the isotropic case, and for the easy-axis and easy-plane cases, $\left(J_{1,2, N N}^{z}-J_{1,2, N N}\right) / J_{1,2, N N}= \pm 0.1$. One can see that the weak magnetic anisotropy of any sign (easy-axis or easy-plane) does not produce drastic differences, comparing to the isotropic case.

\section{Concluding remarks}

In conclusion, motivated by recent experiments on quasi-one-dimensional quantum spin systems, we have studied the one-dimensional model, in which the alternation of the exchange interactions between neighboring spins is accompanied by the next-nearest neighbor spin exchange (zig-zag spin ladder with the alternation). The model permitted to obtain thermodynamic characteristics of the considered quantum spin chain in the mean-field like approximation. The most important behavior of the model is in the ground state. Depending on the strength of the NNN interactions, the model manifests either the spin-gapped behavior of low-lying excitations at low magnetic fields, or the ground state ferrimagnetic ordering with gapless low-lying excitations, or the incommensurate magnetic phase. The system undergoes second order or first order quantum phase transitions, governed by the external magnetic field, NNN coupling strength (the later can be caused by an external or internal pressure), and the degree of the alternation. Hence, on the one hand, NNN spin-spin interactions in a dimerized quantum spin chain can produce a spontaneous magnetization. On the other hand, for quantum spin chains with a spontaneous magnetization, caused by NNN spin-spin couplings, the alternation of $\mathrm{NN}$ exchange interactions can be the reason for destroying of that magnetization and the onset of a spin gap for low-lying excitations. The weak uniaxial magnetic anisotropy does not affect the main features of the thermodynamic characteristics of the spin chain (zig-zag ladder). Alternating NN interactions produce a spin gap between two branches of low-energy excitations, and the NNN interactions yield asymmetry of dispersion laws of those excitations, with possible minima, corresponding to incommensurate structures in the chain. It is interesting to notice that two branches of magnetic excitations, with the lowest branch having minima at incommensurate wave vectors, cf. Figs. 4 and 5, were recently observed in inelastic neutron scattering experiments on a quasi-one dimensional compound $\mathrm{Li}_{2} \mathrm{CuO}_{2}$ at temperatures, higher than the Néel temperature [25]. In that com- pound it is believed that intra-chain ferromagnetic NN coupling between spins is accompanied by the antiferromagnetic NNN coupling.

Our numerical exact diagonalization results have shown that the mean field like approximation correctly reproduces the features of the behavior of spin chains with alternating nearest neighbor and next-nearest neighbor interactions for the ferromagnetic NN couplings and alternating in sign NN exchange constants, if the strength of the NNN interaction is weak enough. On the other hand, for large values of the NNN coupling, especially for antiferromagnetic interactions in the chain, the mean field apprioximation cannot reproduce the important features of the behavior of such spin chains (zig-zag ladders) in high magnetic fields, which produce additional bands of excitations, and, as a consequence, additional quantum phase transitions in the magnetic field.

A.A.Z. thanks S.-L. Drechsler and W.E.A. Lorenz for interesting discussions. The financial support from the DFG via Grant BU 887/7-1 (A.A.Z.), and the Ukrainian Fundamental Research State Fund via Grant F25.4/13 is acknowledged.

1. A.A. Zvyagin, Finite Size Effects in Correlated Electron Models: Exact Results, Imperial College Press, London (2005).

2. N.D. Mermin and H. Wagner, Phys. Rev. Lett. 17, 1133 (1966).

3. A. Zheludev, M. Kenzelmann, S. Raymond, E. Ressouche, T. Masuda, K. Kakurai, S. Maslov, I. Tsukada, K. Uchinokura, and A. Wildes, Phys. Rev. Lett. 85, 4799 (2000); I. Tsukada, J. Takeya, T. Masuda, and K. Uchinokura, Phys. Rev. B62, R6061 (2000); M. Kohgi, K. Iwasa, J.M. Mignot, B. Fak, P. Gegenwart, M. Lang, A. Ochiai, H. Aoki, and T. Suzuki, Phys. Rev. Lett. 86, 2439 (2001).

4. T. Imai, K.R. Thurber, K.M. Shen, A.W. Hunt, and F.C. Chou, Phys. Rev. Lett. 81, 220 (1998); R.S. Eccleston, M. Uehara, J. Akimitsu, H. Eisaki, N. Motoyama, and S.I. Uchida, ibid. 81, 1702 (1998); M. Windt, M. Grüninger, T. Nunner, C. Knetter, K.P. Schmidt, G.S. Uhrig, T. Kopp, A. Freimuth, U. Ammerahl, B. Büchner, and A. Revcolevschi, ibid. 87, 127002 (2001); K. Magishi, S. Matsumoto, Y. Kitaoka, K. Ishida, K. Asayama, M. Uehara, T. Nagata, and J. Akimitsu, Phys. Rev. B57, 11533 (1998); S.-L. Drechsler, O. Volkova, A.N. Vasiliev, N. Tristan, J. Richter, M. Schmitt, H. Rosner, J. Malek, R. Klingeler, A.A. Zvyagin, and B. Büchner, Phys. Rev. Lett. 98, 077202 (2007); A. Möller, T. Taetz, N. Hollmann, J.A. Mydosh, V. Kataev, M. Yehia, E. Vavilova, and B. Büchner, Phys. Rev. B76, 134411 (2007); Y. Singh, R.W. McCallum, and D.C. Johnston, Phys. Rev. B76, 174402 (2007); S.A.J. Kimber, M.A. de Vries, J. Sanchez-Benitez, K.V. Kamenev, and J.P. Attfield, Phys. Rev. B77, 014428 (2008).

5. G. Misguich, B. Bernu, C. Lhuillier, and C. Waldtmann, Phys. Rev. Lett. 81, 1098 (1998).

6. A.M. Tsvelik, Phys. Rev. B42, 779 (1990); H. Frahm, J. Phys. A25, 1417 (1992). 
7. A.E. Borovik, A.A. Zvyagin, V.Yu. Popkov, and Yu.M. Strzhemechny, Pisma Zh. Eksp. Teor. Fiz. 55, 292 (1992) [JETP Letters 55, 292 (1992)]; A.A. Zvyagin, Fiz. Nizk. Temp. 18, 1029 (1992) [Sov. J. Low Temp. Phys. 18, 723 (1992)]; A.A. Zvyagin, Phys. Rev. Lett. 82, 2409 (1999).

8. V.Yu. Popkov and A.A. Zvyagin, Phys. Lett. A175, 295 (1993); A.A. Zvyagin, Pis'ma Zh. Eksp. Teor. Fiz. 60, 563 (1994) [JETP Letters 60, 580 (1994)]; A.A. Zvyagin, Phys. Rev. B51, 12579 (1995); A.A. Zvyagin, Phys. Rev. B52, 15050 (1995); A.A. Zvyagin, Pis'ma Zh. Eksp. Teor. Fiz. 63, 192 (1996) [JETP Letters 63, 204 (1996)]; A.A. Zvyagin, Phys. Rev. B57, 1035 (1998); A.A. Zvyagin, Fiz. Nizk. Temp. 26, 181 (2000) [Low Temp. Phys. 26, 134 (2000)];

9. N. Muramoto and M. Takahashi, J. Phys. Soc. Jpn. 68, 2098 (1999).

10. A.A. Zvyagin, J. Phys. A34, R21 (2001).

11. A.A. Zvyagin, A. Klümper, and J. Zittarz, Eur. Phys. J. B19, 25 (2001).

12. A.A. Zvyagin and A. Klümper, Phys. Rev. B68, 144426 (2003); A.A. Zvyagin, Phys. Rev. B72, 064419 (2005).

13. C.K. Majumdar and D.K. Ghosh, J. Math. Phys. 10, 1388 (1969).

14. T. Masuda, A. Zheludev, A. Bush, M. Markina, and A. Vasiliev, Phys. Rev. Lett. 92, 177201 (2004); ibid. 94, 039706 (2005); S.-L. Drechsler, J. Málek, J. Richter, A.S. Moskvin, A.A. Gippius, and H. Rosner, Phys. Rev. Lett. 94, 039705 (2005); A.A. Gippius, E.N. Morozova, A.S. Moskvin, A.V. Zalessky, A.A. Bush, M. Baenitz, H. Rosner, and S.-L. Drechsler, Phys. Rev. B70, 020406(R) (2004); H.-A. Krug von Nidda, L.E. Svistov, M.V. Eremin, R.M. Eremina, A. Loidl, V. Kataev, A. Validov, A. Prokofiev, and W. A $\beta$ mus, Phys.
Rev. B65, 134445 (2002); L. Capogna, M. Mayr, P. Horsch, M. Raichle, R.K. Kremer, M. Sofin, A. Maljuk, M. Jansen, and B. Keimer, Phys. Rev. B71, 140402(R) (2005).

15. V. Gnezdilov, P. Lemmens, A.A. Zvyagin, V.O. Cheranovskii, K. Lamonova, Yu.G. Pashkevich, R.K. Kremer, and H. Berger, Phys. Rev. B78, 184407 (2008).

16. W. Geertsma and D. Khomskii, ArXiv:cond-mat/0007421 (unpublished), and references therein.

17. L.N. Bulaevskii, Zh. Eksp. Teor. Fiz. 43, 968 (1962) [Sov. Phys. JETP 16, 685 (1963)].

18. W. Brenig, Phys. Rev. B56, 2551 (1997); T. Verkholyak, A. Honecker, and W. Brenig, Eur. Phys. J. B49, 283 (2006); L. Sun, J. Dai, S. Qin, and J. Zhang, Phys. Lett. A294, 239 (2002).

19. D.V. Dmitriev and V.Ya. Krivnov, Phys. Rev. B73, 024402 (2006); D.V. Dmitriev and V.Ya. Krivnov, ibid. 77, 024401 (2008).

20. L.N. Bulaevskii, Fiz. Tverd. Tela 11, 1132 (1969) [Sov. Phys. Solid State 11, 921 (1969)].

21. D.V. Dmitriev, V.Ya. Krivnov, and J. Richter, Phys. Rev. B75, 014424 (2007).

22. D.V. Dmitriev, V.Ya. Krivnov, and A.A. Ovchinnikov, $Z$. Phys. B103, 193 (1997); D.V. Dmitriev, V.Ya. Krivnov, and A.A. Ovchinnikov, Phys. Rev. B56, 5985 (1997).

23. H. Suzuki and K. Takano, J. Phys. Soc. Jpn. 77, 113701 (2008).

24. A.A. Zvyagin, and G.A. Skorobagatko, Phys. Rev. B73, 024427 (2006).

25. W.E.A. Lorenz, R.O. Kuzian, S.-L- Drechsler, J. Richter, J. Malek, H. Rosner, M. Loewenhaupt, W.D. Stein, A. Hiess, W. Schmidt, N. Wizent, R. Klingeler, and B. Büchner, unpublished. 